\title{
RC beams damaged by cracking and strengthened with NSM CFRP/GFRP rods
}

\author{
R. Capozucca, E. Magagnini, M.V. Vecchietti \\ Struct.Section DICEA, Università Politecnica delle Marche, Ancona, Italy \\ r.capozucca@staff.univpm.it_e.magagnini@staff.univpm.it,m.v.veccbietti@pm.univpm.it \\ S. Khatir \\ Soete Laboratory, Faculty of Engineering and Architecture, Ghent University, Belgium \\ samir.khatin@ugent.be
}

\begin{abstract}
The near surface mounted (NSM) method of inserting fiber reinforced polymer (FRP) elements (rods or lamina) into notches has been shown to be a good way for restoring reinforced concrete $(\mathrm{RC})$ elements. The knowledge about the use of Glass-FRP rod following the NSM to reinforce RC beams is limited. This paper deals with the analysis of static and dynamic behaviour of RC beams with and without strengthening. The response of $\mathrm{RC}$ beams was assessed at different concrete's damage level by non-destructive vibration tests. First, a couple of beams have been analysed: one RC beam subjected to bending and under vibration tests; another one beam, damaged by bending and strengthened with NSM Carbon-FRP rods tested again under vibration. Further, one RC beam damage was analysed under bending and vibration tests without strengthening; successively, the beam model with NSM GFRP rod has been tested following the same loading path. Below experimental results are shown and commented; in particular, changes in frequency values are related to the evolution of damage level affected RC beams with NSM CFRP and GFRP rods.
\end{abstract}

KEYwords. NSM, CFRP/GFRP, Damage, Bending and Vibration tests, Frequency.

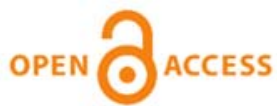

Citation: Capozucca, R., Khatir, S., Magagnini, E., Vecchietti, M.V., RC beams damaged by cracking and strengthened with NSM CFRP/GFRP rods, Frattura ed Integrità Strutturale, 58 (2021) 386-401..

Received: 26.08 .2021

Accepted: 02.09.2021

Published: 01.10.2021

Copyright: (C) 2021 This is an open access article under the terms of the CC-BY 4.0, which permits unrestricted use, distribution, and reproduction in any medium, provided the original author and source are credited.
\end{abstract}

\section{INTRODUCTION}

$\mathrm{T}$ he near surface mounted (NSM) technique for the strengthening of reinforced concrete (RC) elements foresees grooves along the concrete cover where the fibre reinforced polymer (FRP) rods are glued with the use of mortar or epoxy resin. 
The advantages of using NSM FRP rods as strengthening compared to external bounded (EB) FRP strips are several: greater simplicity of installation; more remarkable ability to prevent loss of bond; and above all, minor susceptibility to damage deriving from collision, high temperature and fire [1-3]. Although the NSM technique appears capable of solving several aspects related to the strengthening with composite materials, the current knowledge on NSM strengthening is more limited than EB method.

The effectiveness of NSM FRP rods for strengthening is contingent on preserving the rod-to-concrete bond [4-7]. In fact, bond behaviour has an influence on the ultimate capacity of reinforced elements as well as on serviceability aspects such as crack width and spacing [8-12].

Experimental results of pull-out tests [12-13] show that the filler used to fill the grooves and its properties greatly influences the bond behaviour. The response of the NSM FRP bars in terms of load carrying capacity increases if a filler able to give a better redistribution of the bond stresses along the anchor length is adopted. The most common filler used for the NSM technique is a bi-component epoxy resin [4,5-14]. Experimental data show that the tensile strength values of the epoxy resin can vary between 13.8 and $42.6 \mathrm{MPa}$, while those of cement mortar between 6.3 and $9 \mathrm{MPa}$.

Moreover, some geometrical parameters could affect the adherence and, therefore, the structural behaviour, such as dimensions of the rod section; thickness and height for rectangular section bars; width and depth of the groove; distance between two adjacent grooves; distance between the groove and the edge of the beam [14,15]. Numerical modelling by Finite Element (FE) has proved that the tensile stresses in concrete decrease as the width of the groove increases [14].

Some experimental investigations [15-17] have dealt with assessing the bond behaviour in the case of non-circular FRPs. For rectangular FRP lamina, it is suggested that the minimum width of a groove should not be less than three times the thickness of the rectangular bar and the minimum depth should not be less than 1.5 times the height of the bar itself [18]. Few experimental researches deal with investigating the behaviour of RC beams strengthened with NSM FRP elements made by different composite materials [19-22] and the assessment of strengthened RC beams with NSM FRP rods with non-destructive free vibration tests $[23,24]$.

This paper deals with the investigation by static and dynamic tests on RC beams strengthened both with CFRP and GFRP rods. A couple of beams with one RC beam subjected to bending and under vibration tests at different damage degrees is analyzed, while a second beam, damaged by bending and strengthened with NSM Carbon-FRP rods, has been tested. Another RC beam damaged by bending strengthened by NSM GFRP rod has been experimentally studied. The response of RC beams has been assessed through non-destructive vibration monitoring at different level of damage due to concrete cracking or decrease of bond of FRP rods. Static and vibration results are shown and discussed below.

\section{STATIC AND DYNAMIC TESTS OF RC BEAMS WITH NSM CFRP RODS}

$\mathrm{T}$ wo RC beams, labelled and $\mathrm{B} 0$ and $\mathrm{B} 1$, having a rectangular section of $150 \times 220 \mathrm{~mm}$ and a length of $1700 \mathrm{~mm}$ were subjected to static bending tests. Both beam samples were reinforced with $2+2 \varnothing 10 \mathrm{~mm}$ longitudinal steel bar and shear resistant reinforcement consisting of $\emptyset 6 / 60 \mathrm{~mm}$ stirrups. The reinforcement's entity has been defined to give a scaled behavior with respect to a real beam with greater dimensions; moreover, the stirrup's disposition has been designed to guarantee the failure of the specimens by bending and not by shear. Two notches with dimensions of 20x20 mm were realized at the beam's intrados; the grooves were made for both specimens, but the two $\emptyset 8 \mathrm{~mm}$ CFRP reinforcing bars were inserted only in specimen B1 (Fig. 1) [4]. The beam B0, on the other hand, was tested in the condition without strengthening. Preliminary tests were carried out on concrete, steel and CFRP elements.

Preliminary tests showed that the concrete used has a characteristic cylindrical strength equal to $\mathrm{f}_{\mathrm{c}, \mathrm{av} .} \sim 53.34 \mathrm{~N} / \mathrm{mm}^{2}$ and Young's modulus $\sim 36 \cdot 10^{3} \mathrm{~N} / \mathrm{mm}^{2}$. Monotonic tensile tests were carried out under displacement control on three samples of steel bars, leading to determine an average yielding stress equal to $\mathrm{f}_{\mathrm{y}, \mathrm{av} .} \sim 509 \mathrm{~N} / \mathrm{mm}^{2}$ and Young's modulus about $\sim 2.1 \cdot 105$ $\mathrm{N} / \mathrm{mm}^{2}$. The CFRP rods used have a nominal diameter of $\varnothing 8 \mathrm{~mm}$ and are superficially treated to have better adherence. CFRP rods were tested in tension following the suggestion of [25] and the results are shown in Tab. 1. The average Young's modulus was evaluated equal to $\mathrm{E}_{\mathrm{f}, \mathrm{av}} \sim 1.42 \cdot 105 \mathrm{~N} / \mathrm{mm}^{2}$. A bi-component epoxy resin was adopted to glue the CFRP rods to the concrete.

The behaviour of RC beams with and without FRP NSM strengthening was assessed through four points bending tests, where the two supports and the two loading points were placed, respectively, at $1500 \mathrm{~mm}$ and $300 \mathrm{~mm}$ form the centerline. The static tests were carried out, on all the specimens, by means of loading and unloading cycles and, successively, to increased bending load until failure (Tab. 2). 

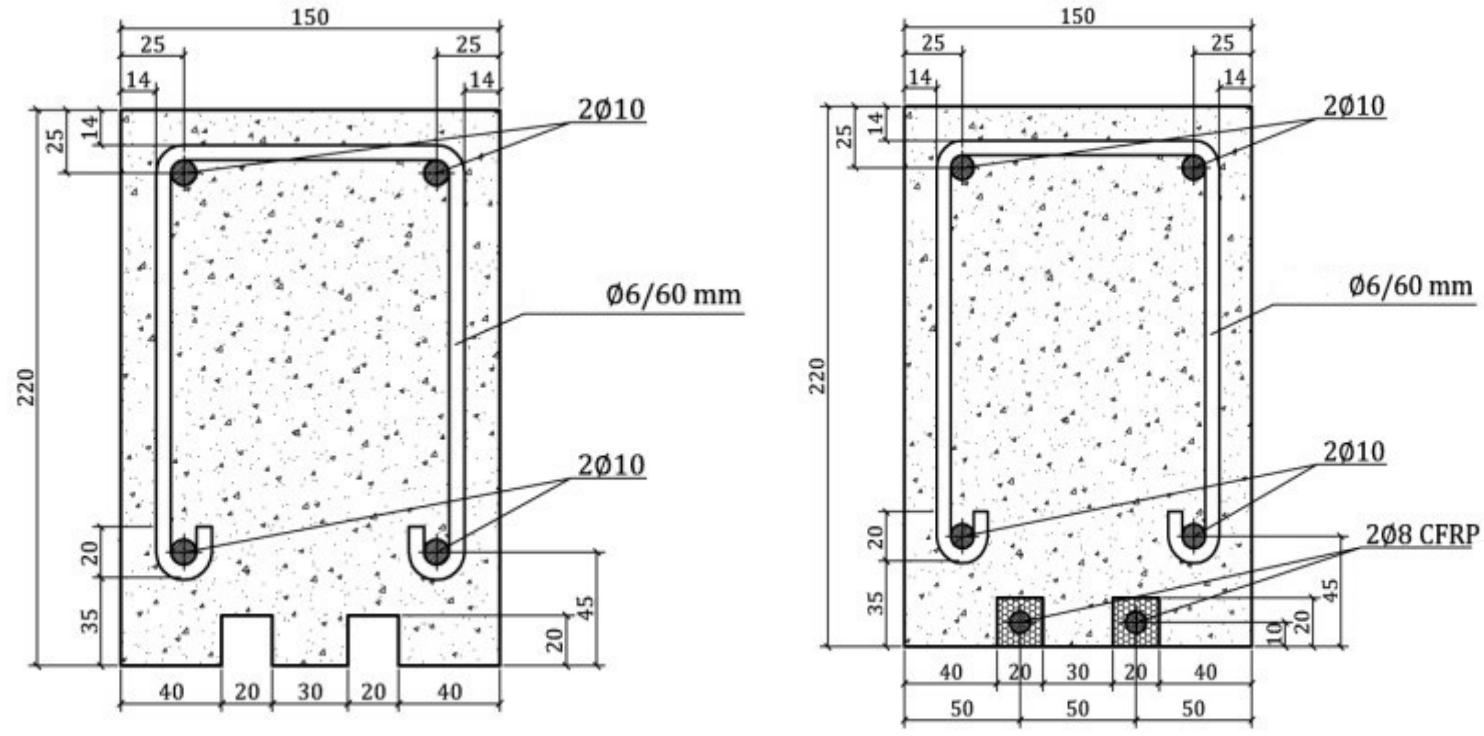

B0

Figure 1: RC beam section with and without CFRP rods.

\begin{tabular}{|c|c|c|c|c|c|c|}
\hline Specimen & $\begin{array}{c}\text { Nominal } \\
\text { Diameter } \\
\varnothing \\
{\left[\mathrm{mm}^{2}\right]}\end{array}$ & $\begin{array}{c}\text { Real } \\
\text { Diameter } \\
\varnothing \\
{\left[\mathrm{mm}^{2}\right]}\end{array}$ & $\begin{array}{c}\text { Section } \\
\text { Area } \\
\text { A } \\
{\left[\mathrm{mm}^{2}\right]}\end{array}$ & $\begin{array}{c}\text { Failure } \\
\text { Load } \\
\mathrm{F}_{\mathrm{m}} \\
{[\mathrm{N}]}\end{array}$ & $\begin{array}{c}\text { Tensile } \\
\text { Strength } \\
f_{t} \\
{\left[\mathrm{~N} / \mathrm{mm}^{2}\right]}\end{array}$ & $\begin{array}{c}\text { Average } \\
\text { Tensile strength } \\
f_{t, a v .} \\
{\left[\mathrm{N} / \mathrm{mm}^{2}\right]}\end{array}$ \\
\hline 1 & 8 & 9.1 & 65.04 & 144270 & 2218.21 & \\
\hline 2 & 8 & 9.1 & 65.04 & 135870 & 2089.06 & \\
\hline 3 & 8 & 9.1 & 65.04 & 134490 & 2067.84 & 2153.27 \\
\hline 4 & 8 & 9.1 & 65.04 & 137200 & 2109.51 & \\
\hline 5 & 8 & 9.1 & 65.04 & 148400 & 2281.71 & \\
\hline
\end{tabular}

Table 1: Results of uniaxial tensile test on CFRP rods.

With the aim to obtain information about the strain's evolution in the steel bars and in the CFRP rod, three electronical strain gauges were adopted; specifically, two of them were applied on the steel longitudinal reinforcement, both positioned at the centerline, one on the beam's extrados and the other one on the intrados; the last one was positioned in the middle of the beam on one of the two CFRP rods. Two horizontal LVDT's recorded the concrete's deformations in the compressed zone. An inductive LVDT with a full scale of $100 \mathrm{~mm}$ and a sensitivity of $0.01 \mathrm{~mm}$ was used to evaluate the beam's deflection at the centerline. Another displacement transducer was also positioned at $100 \mathrm{~mm}$ from the support. A hydraulic jack with a maximum capacity of $500 \mathrm{kN}$ together with a load distribution's system was utilized for the application of the two forces symmetrically applied in the center line at a wheelbase of $300 \mathrm{~mm}$. For each step of cyclic loading a corresponding damage level, identified as Di with $\mathrm{i}=1, \ldots, 7$, was defined. From laboratory static tests, on specimens B0 (without strengthening) and B1 (strengthened with CFRP rods), the experimental results shown in Tab. 3 and Tab. 4 were obtained. The comparison between the envelopes of experimental diagrams moment, M, vs curvature, $\chi$, for beams B0 and B1 is given in Fig. 2. 


\begin{tabular}{ccccc}
\hline & \multicolumn{3}{c}{ Beam B0 } & \multicolumn{2}{c}{ Beam B1 with NSM CFRP rod } \\
Damage degree & Moment $[\mathrm{kNm}]$ & Load $[\mathrm{kN}]$ & Moment $[\mathrm{kNm}]$ & Load $[\mathrm{kN}]$ \\
D1 & 2.16 & 7.21 & 2.13 & 7.10 \\
D2 & 4.28 & 14.32 & 4.29 & 14.29 \\
D3 & 9.46 & 31.50 & 9.48 & 31.61 \\
D4 & 11.61 & 38.73 & 11.63 & 38.77 \\
D5 & 14.30 & 47.80 & 26.39 & 87.97 \\
D6 & - & - & 31.48 & 104.92 \\
D7 & - & - & 34.62 & 115.39 \\
\hline
\end{tabular}

Table 2: Experimental step of loading.

\begin{tabular}{cccccc}
\hline & $\begin{array}{c}\text { Deflection } \delta \\
{[\mathrm{mm}]}\end{array}$ & $\begin{array}{c}\text { Concrete strain } \\
\varepsilon_{\mathrm{c} \text { sup }}\end{array}$ & $\begin{array}{c}\text { Steel strain } \\
\text { extradoss } \\
\varepsilon_{\mathrm{s} 1}\end{array}$ & $\begin{array}{c}\text { Steel strain } \\
\text { intradoss } \\
\varepsilon_{\mathrm{s} 2}\end{array}$ & $\begin{array}{c}\text { Curvature } \\
\chi \\
{\left[1 / \mathrm{mm} \cdot 10^{-6}\right]}\end{array}$ \\
D1 & 0.56 & -0.000203 & -0.000136 & 0.000660 & 5.31 \\
D2 & 1.79 & -0.000494 & -0.000181 & 0.001092 & 18.41 \\
D3 & 5.07 & -0.001011 & -0.000362 & 0.002399 & 32.2 \\
D4 & 8.62 & -0.001796 & -0.000154 & 0.004676 & - \\
D5 & 28.02 & - & - & - & \\
\hline
\end{tabular}

Table 3: Experimental data obtained by static tests for beam B0.

\begin{tabular}{ccccccc}
\hline & $\begin{array}{c}\text { Deflection } \delta \\
{[\mathrm{mm}]}\end{array}$ & $\begin{array}{c}\text { Concrete } \\
\text { strain } \\
\varepsilon_{\mathrm{c}, \mathrm{sup}}\end{array}$ & $\begin{array}{c}\text { Steel strain } \\
\text { extradoss } \\
\varepsilon_{\mathrm{s} 1}\end{array}$ & $\begin{array}{c}\text { Steel strain } \\
\text { intradoss } \\
\varepsilon_{\mathrm{s} 2}\end{array}$ & $\begin{array}{c}\text { CFRP } \\
\text { Strain } \\
\varepsilon_{\mathrm{cfrp}}\end{array}$ & $\begin{array}{c}\text { Curvature } \\
\chi \\
{\left[1 / \mathrm{mm} 10^{-6}\right]}\end{array}$ \\
D1 & 0.25 & -0.000070 & -0.000096 & 0.000034 & 0.000093 & 1.02 \\
D2 & 0.67 & -0.000171 & -0.000186 & 0.000238 & 0.000329 & 2.78 \\
D3 & 2.17 & -0.000421 & -0.000422 & 0.000925 & 0.001373 & 9.70 \\
D4 & 2.88 & -0.000530 & -0.000510 & 0.001231 & 0.001767 & 12.31 \\
D5 & 7.17 & -0.001208 & -0.001039 & 0.002873 & 0.004151 & 28.05 \\
D6 & 10.66 & -0.001690 & -0.001290 & 0.002764 & 0.005748 & 38.04 \\
D7 & 15.25 & -0.001997 & -0.001459 & 0.002836 & 0.008690 & 54.86 \\
\hline
\end{tabular}

Table 4: Experimental data obtained by static tests for beam B1. 
The experimental modal testing was performed adopting the so call "mobile accelerometer" technique where an accelerometer, positioned at several points during tests, measures the beam's acceleration after the excitation by an impact hammer positioned at a fixed point. The fixed point of specimen's excitation by impact was established as the one placed at $4.5 \mathrm{~cm}$ from one end (point CH1 in Fig. 3). The response of the structure is obtained as an average of 10 impacts for each accelerometer position, for a total of 14 accelerometer positions. The accelerometer used for the dynamic experimentation is model 4508 - Piezoelectric CCLD accelerometer, $100 \mathrm{mV} / \mathrm{g}, 1$ slot, top connector, by Brüel \& Kjær; it is a piezoelectric transducer. This accelerometer model has a very low weight $(4.8 \mathrm{~g})$ and covers a frequency range from 0.3 $\mathrm{Hz}$ to $8000 \mathrm{~Hz}$ with a sensitivity of $10 \mathrm{mV} / \mathrm{g}$.

One of the aims of the dynamic experimental program is to obtain a comparison between the experimental and theoretical characterization of the beam models, to check the reliability of the experimental results. We then proceeded to the theoretical determination of the first four natural frequencies for the specimens with and without CFRP NSM reinforcement according to the Euler-Bernoulli continuous beam model.

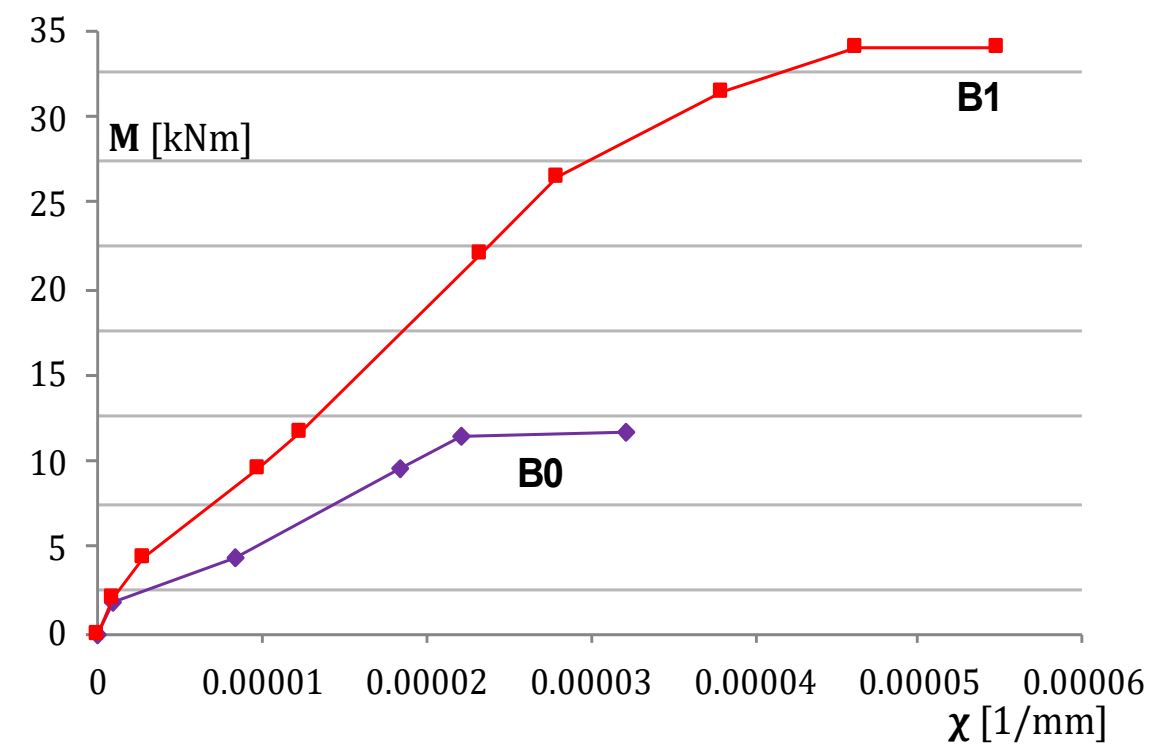

Figure 2: Experimental diagrams bending moment, $\mathrm{M}$, - curvature, $\chi$, beams B0 and B1.

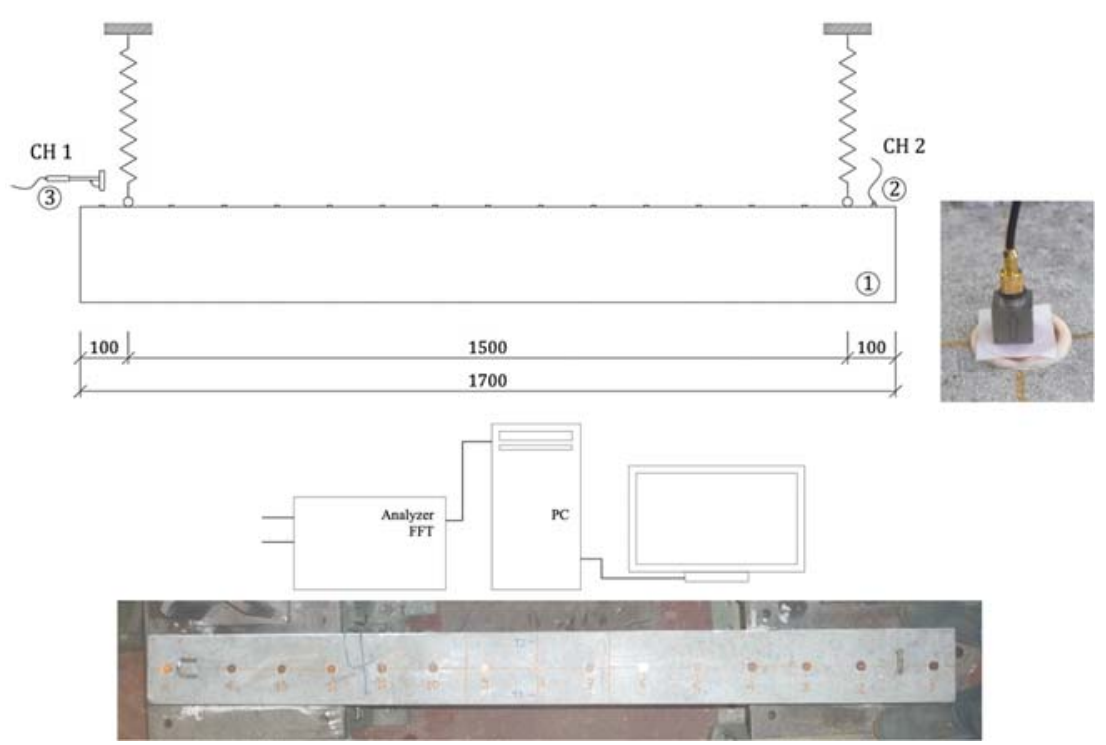

Figure 3: Instrumentations for vibration tests. 
For beam B0, Tab. 5 contains the natural frequencies obtained for each position of the accelerometer at different level of damage. Fig. 4 shows the variations of the experimental frequencies with reference to the damage-free state (D0) for cracking due to the increase in bending; the variation of the experimental frequencies with respect to D0, for all damage levels, for the first four vibration modes, is expressed as:

$$
\frac{\Delta f_{r}}{f_{D 0}}=100 \frac{f_{D 0}-f_{D i}}{f_{D 0}}
$$

where $f_{D i}$ is the frequency obtained for the damage condition Di and $f_{D 0}$ is the frequency obtained in the undamaged condition D0.

For beam B1, Tab. 6 summarized the natural frequencies obtained for each position of the accelerometer at different level of damage. The variations of the experimental frequencies with reference to the damage-free state (D0) for cracking due to the increase in bending are shown in Fig. 5.

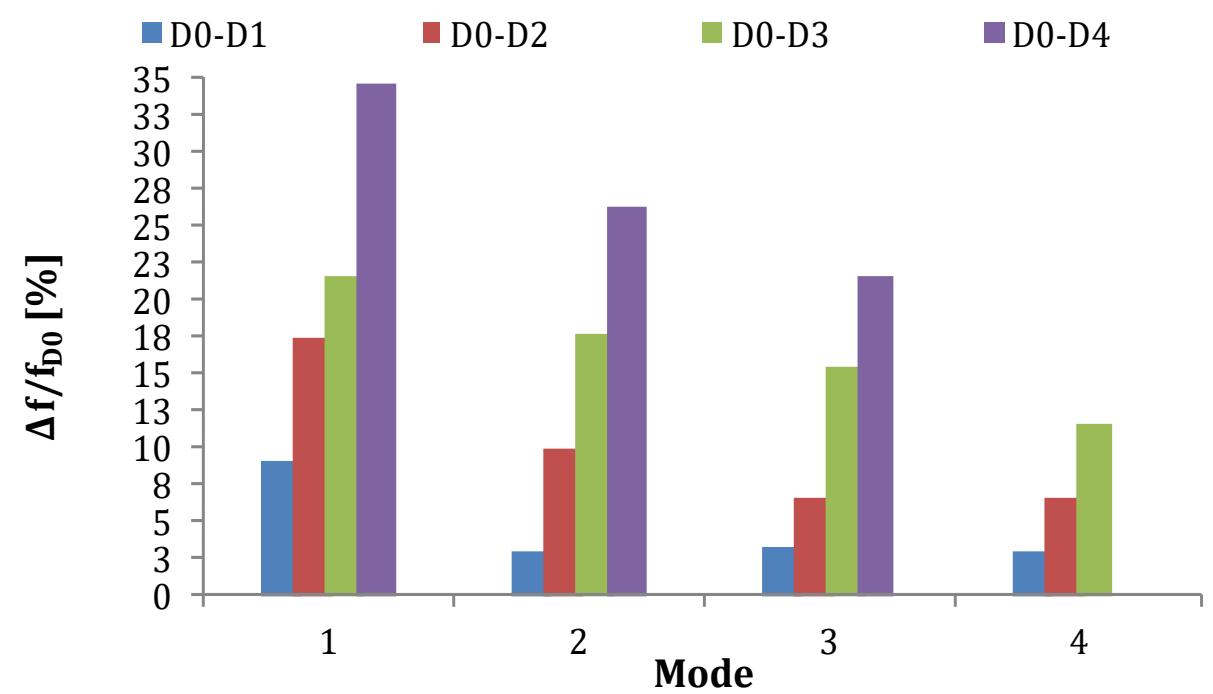

Figure 4: Variation percent of frequencies for beam B0 at different damage degree.

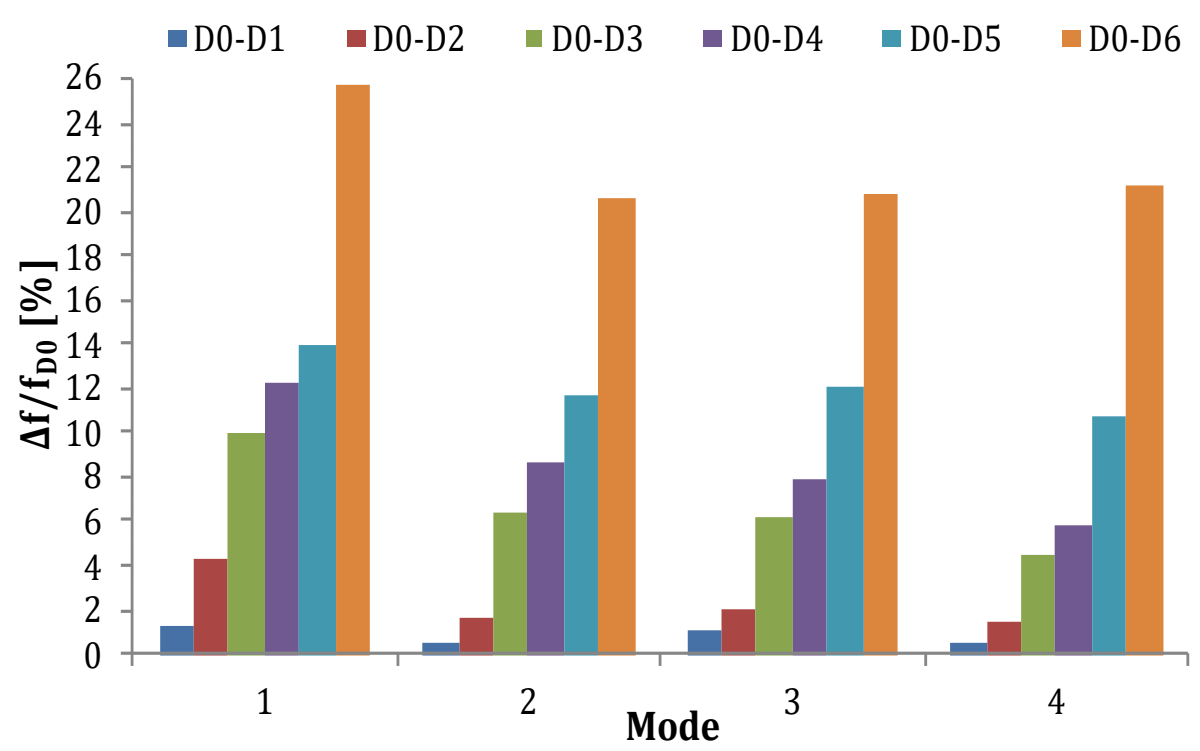

Figure 5: Variation percent of frequencies values for beam B1 at different damage degree. 


\begin{tabular}{|c|c|c|c|c|c|c|c|c|c|c|c|c|}
\hline \multicolumn{7}{|c|}{ MODE 1} & \multicolumn{6}{|c|}{ MODE 2} \\
\hline \multicolumn{7}{|c|}{ Frequency $[\mathrm{Hz}]$} & \multicolumn{6}{|c|}{ Frequency $[\mathrm{Hz}]$} \\
\hline & Theor. & D0 & D1 & $\mathrm{D} 2$ & D3 & D4 & Theor. & D0 & D1 & $\mathrm{D} 2$ & D3 & D4 \\
\hline M1 & 308 & 275 & 252 & 228 & 215 & 180 & 848 & 706 & 686 & 635 & 579 & 519 \\
\hline M2 & 308 & 275 & 251 & 227 & 215 & 180 & 848 & 706 & 686 & 635 & 580 & 521 \\
\hline M3 & 308 & 276 & 251 & 226 & 215 & 180 & 848 & 706 & 685 & 635 & 579 & 521 \\
\hline M4 & 308 & 275 & 250 & 228 & 215 & 180 & 848 & 706 & 685 & 635 & 579 & 520 \\
\hline M5 & 308 & 275 & 250 & 228 & 216 & 180 & 848 & 706 & 685 & 635 & 580 & 521 \\
\hline M6 & 308 & 275 & 250 & 227 & 216 & 180 & 848 & 706 & 684 & 634 & 580 & 520 \\
\hline M7 & 308 & 275 & 249 & 228 & 216 & 180 & 848 & 706 & 684 & 635 & 580 & 521 \\
\hline M8 & 308 & 275 & 250 & 228 & 216 & 180 & 848 & - & - & - & - & - \\
\hline M9 & 308 & 275 & 249 & 228 & 216 & 180 & 848 & 706 & 684 & 636 & 581 & 520 \\
\hline M10 & 308 & 275 & 249 & 227 & 216 & 180 & 848 & 706 & 684 & 635 & 581 & 520 \\
\hline M11 & 308 & 275 & 249 & 226 & 216 & 180 & 848 & 706 & 684 & 635 & 581 & 520 \\
\hline M12 & 308 & 275 & 250 & 226 & 216 & 180 & 848 & 706 & 684 & 635 & 581 & 521 \\
\hline M13 & 308 & 275 & 249 & 228 & 216 & 180 & 848 & 706 & 684 & 635 & 581 & 522 \\
\hline M14 & 308 & 275 & 250 & 228 & 217 & 180 & 848 & 706 & 684 & 635 & 582 & 520 \\
\hline average & 308 & 275 & 250 & 227 & 216 & 180 & 848 & 706 & 685 & 635 & 580 & 520 \\
\hline
\end{tabular}

\begin{tabular}{|c|c|c|c|c|c|c|c|c|c|c|c|c|}
\hline \multicolumn{7}{|c|}{ MODE 3} & \multicolumn{6}{|c|}{ MODE 4} \\
\hline \multicolumn{7}{|c|}{ Frequency $[\mathrm{Hz}]$} & \multicolumn{6}{|c|}{ Frequency [Hz] } \\
\hline & Theor. & D0 & D1 & D2 & D3 & D4 & Theor. & D0 & D1 & $\mathrm{D} 2$ & D3 & D4 \\
\hline M1 & 1663 & 1274 & 1238 & 1192 & 1076 & 999 & 2749 & 1875 & 1823 & 1753 & 1657 & - \\
\hline M2 & 1663 & - & 1237 & 1149 & 1079 & 999 & 2749 & - & 1824 & 1753 & 1658 & - \\
\hline M3 & 1663 & - & 1237 & 1193 & 1077 & 1000 & 2749 & - & 1822 & 1752 & 1659 & - \\
\hline M4 & 1663 & 1274 & 1235 & 1193 & 1075 & 999 & 2749 & - & 1822 & 1751 & 1658 & - \\
\hline M5 & 1663 & 1274 & 1235 & 1194 & 1076 & 999 & 2749 & - & 1823 & 1754 & 1662 & - \\
\hline M6 & 1663 & - & 1233 & - & 1079 & 1000 & 2749 & 1881 & 1822 & 1751 & 1662 & - \\
\hline M7 & 1663 & 1274 & 1233 & 1195 & 1077 & 1000 & 2749 & - & 1824 & 1755 & 1662 & - \\
\hline M8 & 1663 & 1274 & 1233 & 1195 & 1077 & 1000 & 2749 & - & - & - & - & - \\
\hline M9 & 1663 & 1274 & 1233 & 1195 & 1079 & 999 & 2749 & 1878 & 1821 & 1755 & 1663 & - \\
\hline M10 & 1663 & 1274 & - & - & - & - & 2749 & 1879 & 1823 & 1753 & 1664 & - \\
\hline M11 & 1663 & 1275 & 1233 & 1193 & 1080 & 1000 & 2749 & $\mathrm{NC}$ & 1824 & 1753 & $\mathrm{NC}$ & - \\
\hline M12 & 1663 & 1275 & 1232 & 1193 & 1080 & 1001 & 2749 & 1878 & 1820 & 1753 & 1665 & - \\
\hline M13 & 1663 & 1274 & 1232 & 1196 & 1079 & 1001 & 2749 & 1877 & 1816 & 1753 & 1664 & - \\
\hline M14 & 1663 & 1275 & 1231 & 1196 & 1084 & - & 2749 & $\mathrm{NC}$ & 1820 & 1755 & - & - \\
\hline average & 1663 & 1274 & 1234 & 1190 & 1078 & 1000 & 2749 & 1878 & 1822 & 1753 & 1661 & - \\
\hline
\end{tabular}

Table 5: Average experimental frequency values recorded for all mark points Mi with i=1,..,14 at different damage degree on beam B0. 


\begin{tabular}{|c|c|c|c|c|c|c|c|c|c|c|c|c|}
\hline \multicolumn{7}{|c|}{ MODE 1} & \multicolumn{6}{|c|}{ MODE 2} \\
\hline \multicolumn{7}{|c|}{ Frequency $[\mathrm{Hz}]$} & \multicolumn{6}{|c|}{ Frequency $[\mathrm{Hz}]$} \\
\hline & Theor. & D0 & D1 & D2 & D3 & D4 & Theor. & D0 & D1 & $\mathrm{D} 2$ & D3 & D4 \\
\hline M1 & 311 & 284 & 281 & 272 & 254 & 248 & 857 & 723 & 719 & 710 & 674 & 659 \\
\hline M2 & 311 & 284 & 281 & 272 & 254 & 249 & 857 & 723 & 719 & 710 & 674 & 659 \\
\hline M3 & 311 & 284 & 281 & 272 & 255 & 249 & 857 & 723 & 719 & 710 & 674 & 660 \\
\hline M4 & 311 & 284 & 281 & 272 & 255 & 249 & 857 & 723 & 719 & 710 & 676 & 660 \\
\hline M5 & 311 & 284 & 280 & 272 & 256 & 249 & 857 & 722 & 719 & 710 & 676 & 660 \\
\hline M6 & 311 & 284 & 280 & 272 & 256 & 249 & 857 & 722 & 719 & 710 & 676 & 660 \\
\hline M7 & 311 & 284 & 280 & 272 & 256 & 249 & 857 & 723 & 719 & 711 & 677 & 660 \\
\hline M8 & 311 & 284 & 280 & 272 & 256 & 249 & 857 & - & - & - & - & - \\
\hline M9 & 311 & 284 & 280 & 272 & 256 & 249 & 857 & 722 & 719 & 711 & 676 & 660 \\
\hline M10 & 311 & 284 & 280 & 272 & 256 & 250 & 857 & 722 & 719 & 711 & 677 & 661 \\
\hline M11 & 311 & 284 & 280 & 272 & 256 & 250 & 857 & 722 & 719 & 711 & 677 & 661 \\
\hline M12 & 311 & 284 & 280 & 272 & 256 & 250 & 857 & 723 & 719 & 711 & 677 & 661 \\
\hline M13 & 311 & 284 & 280 & 272 & 257 & 250 & 857 & 723 & 719 & 711 & 677 & 662 \\
\hline M14 & 311 & 284 & 280 & 272 & 256 & 250 & 857 & 722 & 719 & 710 & 677 & 662 \\
\hline average & 311 & 284 & 280 & 272 & 256 & 249 & 857 & 723 & 719 & 710 & 676 & 660 \\
\hline
\end{tabular}

\begin{tabular}{|c|c|c|c|c|c|c|c|c|c|c|c|c|}
\hline \multicolumn{7}{|c|}{ MODE 3} & \multicolumn{6}{|c|}{ MODE 4} \\
\hline \multicolumn{7}{|c|}{ Frequency $[\mathrm{Hz}]$} & \multicolumn{6}{|c|}{ Frequency $[\mathrm{Hz}]$} \\
\hline & Theor. & D0 & D1 & D2 & D3 & D4 & Theor. & D0 & D1 & D2 & D3 & D4 \\
\hline M1 & 1680 & 1298 & 1294 & 1281 & 1216 & 1195 & 2777 & 1923 & 1915 & 1893 & 1833 & 1809 \\
\hline M2 & 1680 & - & 1237 & 1221 & 1216 & 1195 & 2777 & 1923 & 1914 & 1895 & 1835 & 1812 \\
\hline M3 & 1680 & 1298 & 1293 & 1280 & 1216 & 1196 & 2777 & 1923 & 1913 & 1894 & 1835 & 1812 \\
\hline M4 & 1680 & 1298 & 1293 & 1281 & 1218 & 1197 & 2777 & 1922 & 1913 & 1893 & 1835 & 1816 \\
\hline M5 & 1680 & 1299 & 1293 & 1281 & 1218 & 1197 & 2777 & 1924 & 1914 & 1896 & 1837 & 1811 \\
\hline M6 & 1680 & - & 1245 & 1232 & 1220 & 1196 & 2777 & 1923 & 1914 & 1894 & 1838 & 1811 \\
\hline M7 & 1680 & 1299 & 1293 & 1281 & 1219 & 1197 & 2777 & 1924 & 1913 & 1896 & 1839 & 1811 \\
\hline M8 & 1680 & 1299 & 1293 & 1282 & 1219 & 1197 & 2777 & - & - & - & - & - \\
\hline M9 & 1680 & 1298 & 1293 & 1281 & 1219 & 1197 & 2777 & 1922 & 1913 & 1895 & 1839 & 1812 \\
\hline M10 & 1680 & 1300 & 1292 & 1282 & 1219 & 1197 & 2777 & 1922 & 1914 & 1893 & 1839 & 1813 \\
\hline M11 & 1680 & 1301 & 1293 & 1281 & 1219 & 1198 & 2777 & 1923 & 1913 & 1894 & 1840 & 1813 \\
\hline M12 & 1680 & 1298 & 1293 & 1282 & 1219 & 1198 & 2777 & 1922 & 1914 & 1896 & 1839 & 1814 \\
\hline M13 & 1680 & 1299 & 1292 & 1281 & 1219 & 1198 & 2777 & 1922 & 1913 & 1894 & 1839 & 1814 \\
\hline M14 & 1680 & 1301 & 1292 & 1283 & 1219 & 1199 & 2777 & 1924 & 1914 & 1894 & 1840 & 1814 \\
\hline average & 1663 & 1274 & 1234 & 1190 & 1078 & 1000 & 2749 & 1878 & 1822 & 1753 & 1661 & - \\
\hline
\end{tabular}

Table 6: Average experimental frequency values recorded for all mark points Mi with $\mathrm{i}=1, \ldots, 14$ at different damage degree on beam B1. 


\begin{tabular}{|c|c|c|c|c|c|c|c|c|}
\hline & \multirow{2}{*}{$\begin{array}{c}\text { Moment } \\
{[\mathrm{kNm}]}\end{array}$} & \multirow{2}{*}{$\begin{array}{l}\text { Curvature } \\
\chi \\
{\left[1 / \mathrm{mm} \cdot 10^{-6}\right]}\end{array}$} & \multicolumn{2}{|c|}{ MODE 1} & \multicolumn{2}{|c|}{ MODE 2} & \multicolumn{2}{|c|}{ MODE 3} \\
\hline & & & $\Delta \mathrm{f}=\mathrm{f}_{\mathrm{D} 0}-\mathrm{f}_{\mathrm{Di}}$ & $\begin{array}{c}\Delta \mathrm{f} / \mathrm{f}_{\mathrm{D} 0} \\
{[\%]}\end{array}$ & $\Delta \mathrm{f}=\mathrm{f}_{\mathrm{D} 0}-\mathrm{f}_{\mathrm{Di}}$ & $\begin{array}{c}\Delta \mathrm{f} / \mathrm{f}_{\mathrm{D} 0} \\
{[\%]}\end{array}$ & $\Delta \mathrm{f}=\mathrm{f}_{\mathrm{D} 0}-\mathrm{f}_{\mathrm{Di}}$ & $\begin{array}{c}\Delta \mathrm{f} / \mathrm{f}_{\mathrm{D} 0} \\
{[\%]}\end{array}$ \\
\hline D0 & 0 & 0 & D0-D0 & - & D0-D0 & - & D0-D0 & - \\
\hline D1 & 1.803 & 0.0858 & D0-D1 & 9.14 & D0-D1 & 3.04 & D0-D1 & 3.16 \\
\hline D2 & 4.287 & 0.848 & D0-D2 & 17.35 & D0-D2 & 10.06 & D0-D2 & 6.59 \\
\hline D3 & 9.463 & 1.84 & D0-D3 & 21.55 & D0-D3 & 17.80 & D0-D3 & 15.38 \\
\hline D4 & 11.461 & 2.22 & D0-D4 & 34.56 & D0-D4 & 26.28 & D0-D4 & 21.54 \\
\hline D5 & 11.606 & 3.22 & & & & & & \\
\hline
\end{tabular}

Table 7: Exp. values by static test and frequency variations $\Delta \mathrm{f} / \mathrm{fD} 0$ for each damage Di - B0.

\begin{tabular}{|c|c|c|c|c|c|c|c|c|}
\hline & \multirow{2}{*}{$\begin{array}{l}\text { Moment } \\
{[\mathrm{kNm}]}\end{array}$} & \multirow{2}{*}{$\begin{array}{c}\text { Curvature } \\
\chi \\
{\left[1 / \mathrm{mm} \cdot 10^{-6}\right]}\end{array}$} & \multicolumn{2}{|c|}{ MODE 1} & \multicolumn{2}{|c|}{ MODE 2} & \multicolumn{2}{|c|}{ MODE 3} \\
\hline & & & $\Delta \mathrm{f}=\mathrm{f}_{\mathrm{D} 0}-\mathrm{f}_{\mathrm{Di}}$ & $\begin{array}{c}\Delta \mathrm{f} / \mathrm{f}_{\mathrm{D} 0} \\
{[\%]}\end{array}$ & $\Delta \mathrm{f}=\mathrm{f}_{\mathrm{D} 0}-\mathrm{f}_{\mathrm{Di}}$ & $\begin{array}{c}\Delta \mathrm{f} / \mathrm{f}_{\mathrm{D} 0} \\
{[\%]}\end{array}$ & $\Delta \mathrm{f}=\mathrm{f}_{\mathrm{D} 0}-\mathrm{f}_{\mathrm{Di}}$ & $\begin{array}{c}\Delta \mathrm{f} / \mathrm{f}_{\mathrm{D} 0} \\
{[\%]}\end{array}$ \\
\hline D0 & 0 & 0 & D0-D0 & - & D0-D0 & - & D0-D0 & - \\
\hline D1 & 2.1309 & 0.102 & D0-D1 & 1.31 & D0-D1 & 0.49 & D0-D1 & 1.04 \\
\hline D2 & 4.287 & 0.278 & D0-D2 & 4.23 & D0-D2 & 1.67 & D0-D2 & 1.96 \\
\hline D3 & 9.482 & 0.970 & D0-D3 & 9.98 & D0-D3 & 6.44 & D0-D3 & 6.21 \\
\hline D4 & 11.631 & 1.231 & D0-D4 & 12.22 & D0-D4 & 8.60 & D0-D4 & 7.86 \\
\hline D5 & 26.39 & 2.805 & D0-D5 & 13.93 & D0-D5 & 11.75 & D0-D5 & 12.05 \\
\hline D6 & 31.477 & 3.804 & D0-D6 & 25.68 & D0-D6 & 20.56 & D0-D6 & 20.86 \\
\hline D7 & 34.617 & 5.486 & & & & & & \\
\hline
\end{tabular}

Table 8: Exp. values by static test and frequency variations $\Delta \mathrm{f} / \mathrm{fD} 0$ for each damage Di - B1.

Tab. 7 and Tab. 8 summarize the experimental values of frequency variations $\Delta \mathrm{f} / \mathrm{fD} 0$ evaluated for beams B0 and B1 in relation to the curvatures, $\chi$, obtained by static tests at different damage degree Di.

In Fig. 6 the frequency variations in percent for equivalent steps of damage Di in relation to the undamaged state D0 for the first four vibration modes $r=1, \ldots 4$ are compared considering both beam models.

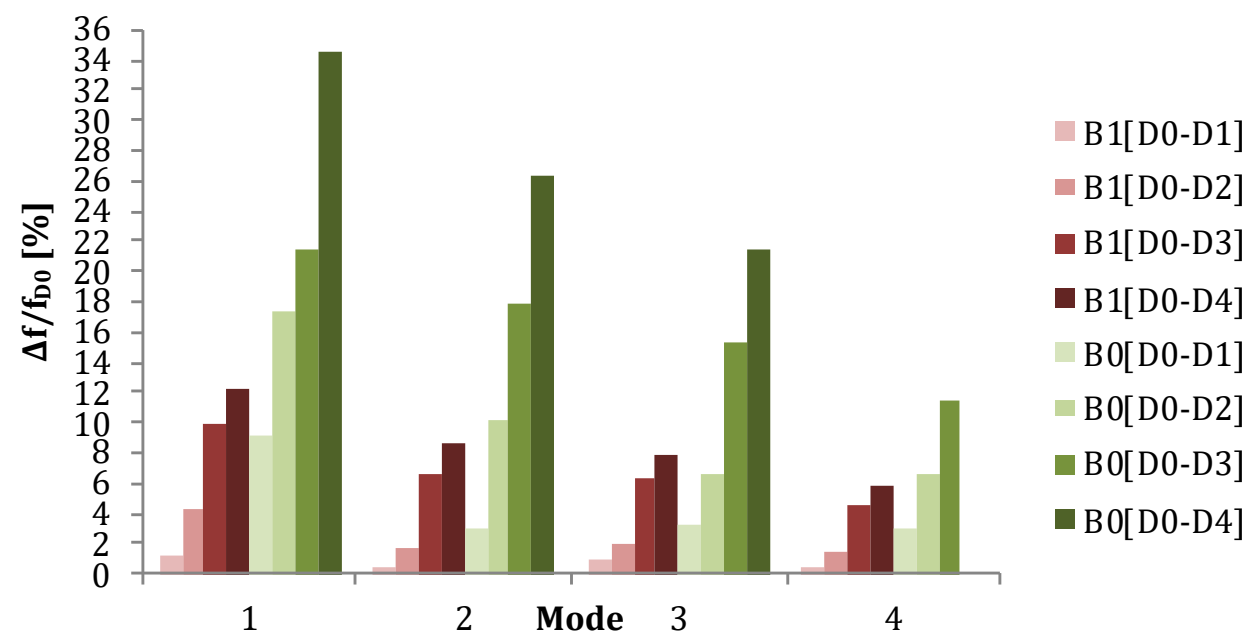

Figure 6: Frequency value variations for equivalent steps of damage Di in relation to the undamaged state D0 for the first four modes $\mathrm{r}=1, \ldots 4$ - beams $\mathrm{B} 0$ and $\mathrm{B} 1$ 


\section{STATIC AND DYNAMIC TESTS OF RC BEAMS WITH NSM GFRP RODS}

T $\mathrm{n}$ this Section, the experimental behaviour of one RC beam, identified as B2, with and without NSM GFRP rod strengthening, is assessed by free vibration consider the undamaged condition and the damaged condition obtained by concrete cracking due to bending.

The RC beam was initially subjected to static tests without strengthening; once obtained a relevant crack patter, the NSM GFRP rod strengthening was applied, and the RC beam was once again subjected to loading.

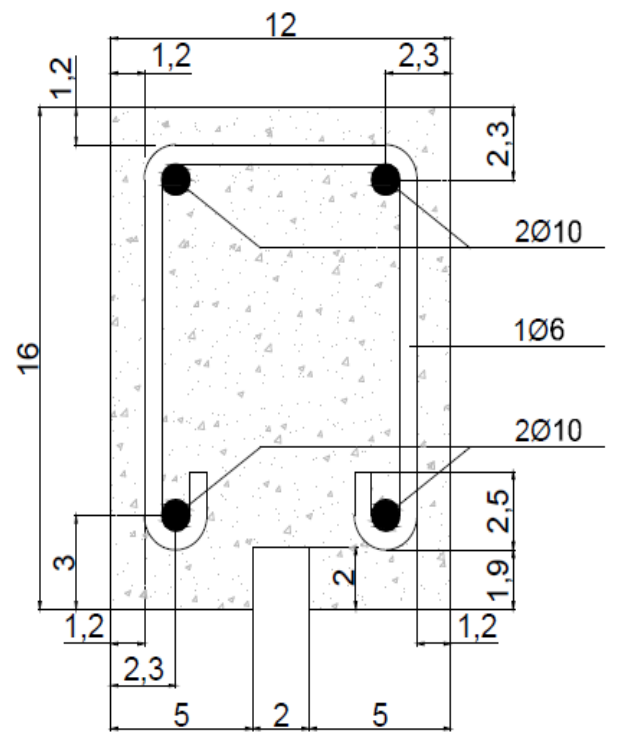

Figure 7: Geometric section of RC beam B2 with steel reinforcement.

\begin{tabular}{ccccccccc}
\hline & Concrete & \multicolumn{3}{c}{ Steel } & & GFRP rod & Epoxy resin \\
$\begin{array}{c}\text { Cylindrical } \\
\text { compressive } \\
\text { strength } \\
{\left[\mathrm{N} / \mathrm{mm}^{2}\right]}\end{array}$ & $\begin{array}{c}\text { Young's } \\
\text { modulus }\end{array}$ & Density & $\begin{array}{c}\text { Yielding } \\
\text { strength }\end{array}$ & $\begin{array}{c}\text { Young's } \\
\text { modulus }\end{array}$ & $\begin{array}{c}\text { Tensile } \\
\text { strength }\end{array}$ & $\begin{array}{c}\text { Young's } \\
\text { modulus }\end{array}$ & $\begin{array}{c}\text { Young's } \\
\text { modulus }\end{array}$ & $\begin{array}{c}\text { Poisson's } \\
\text { coefficient }\end{array}$ \\
44 & $35.0 \cdot 10^{3}$ & $2.4 \cdot 10^{-9}$ & 500 & 34.50 & 1040 & $34 \cdot 10^{3}$ & $1.6 \cdot 10^{3}$ & 0.20 \\
\hline
\end{tabular}

Table 9: Results of preliminary tests on materials.

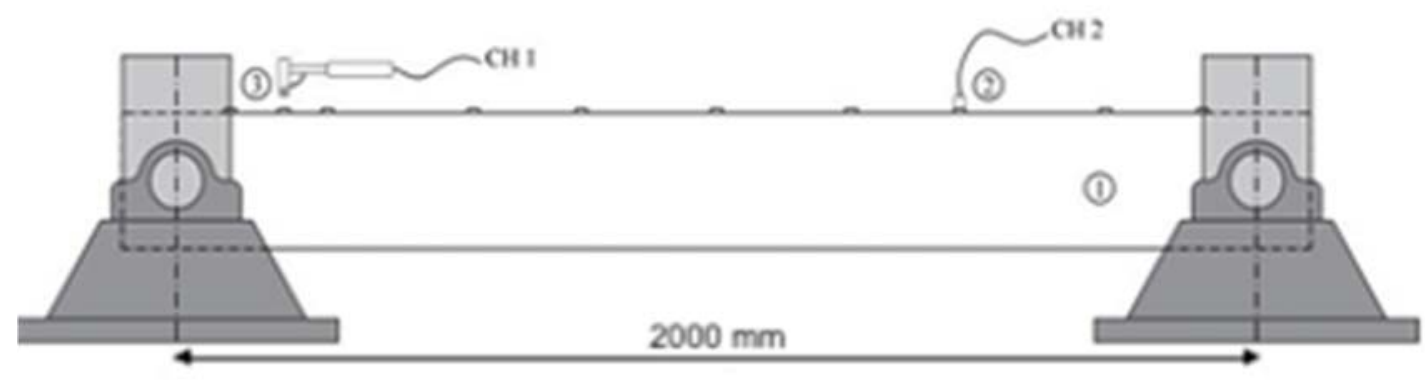

Figure 8: Configuration of modal testing: impact hammer and accelerometer. 
In this phase, the static and dynamic investigations were carried out on a RC beam model characterized by a rectangular section of 120x160 mm and a length of $2200 \mathrm{~mm}$, as shown in Fig. 7. The sample is reinforced with upper and lower longitudinal reinforcement of $2 \varnothing 10$ and shear resistant reinforcement of $\varnothing 6$ stirrups. Also in this case, a $2 \times 2 \mathrm{~cm}$ intradosal groove has been prepared to allow the accommodation of the Ø9-GFRP rod following the NSM technique, after the first phase of testing (Fig. 7). Tab. 9 contains a summary of the mechanical characteristics of each material, as concrete, steel, GFRP rod and epoxy resin, experimentally got by preliminary tests. The mechanical features of the GFRP rod were obtained by laboratory tests on two samples carried out following the ASTM-D 3039 Standard [25].

As already introduced, the experimental characterization of beam B2 involves static and dynamic tests. The hinge ends configuration was adopted for the investigation to reach the condition of simply supported beam. In Fig. 8, it can be observed the apparatus adopted for reproduced the hinge constraint at the ends of beam. This condition was maintained for both static bending and vibration tests. For reproduce the hinge constraints, customized metal devices were realized; 3 $\mathrm{mm}$ metal disk and a $3 \mathrm{~mm}$ thick neoprene lamina were used to solve contact problems at the extremities.

First of all, the undamaged condition of beam B2 without strengthening was tested by vibration. The beam was excited by applying an impulse with a hammer as impact device. The impact was applied in a point maintained fixed during tests (Fig. 8). A response transducer, that is a piezoelectric accelerometer, was adopted for sensing force and motion of the beam. It was placed in 9 different positions and an average of 10 impacts was considered for each location. A Fast Fourier Transformation (FFT) two-channel analyser and PULSE software allowed the data acquisition.

The geometrical and mechanical parameters given above were considered for the evaluation of theoretical frequencies adopting the Euler-Bernoulli beam model. Fig. 9 gives a comparison between the frequency values obtained theoretically and experimentally from free vibration tests on the beam with different constraint conditions at the ends and always in an undamaged condition D0. This comparison allowed us to check the quality of the experimental apparatus, in such a way as to have a control over the reliability of the experimental measurements.

After the first dynamical characterization, RC beam B2 was subjected to a series of load step P by bending. For each phase of loading, different damage conditions Di with $\mathrm{i}=1, \ldots, 3$ due to concrete cracking were identified. Three different cycles of loading were identified: $\mathrm{P} 1=4.0 \mathrm{kN}, \mathrm{P} 2=8.0 \mathrm{kN}, \mathrm{P} 3=18.0 \mathrm{kN}$ (Fig. 10). The bending tests involve the use of vertical hydraulic jack together with a load cell, as system to transfer and measure the applied load; strain gauges, as electronic devices to measure the strain on the steel reinforcement; two LVDTs, as mechanical devises to record deflection at the centerline and near the support.

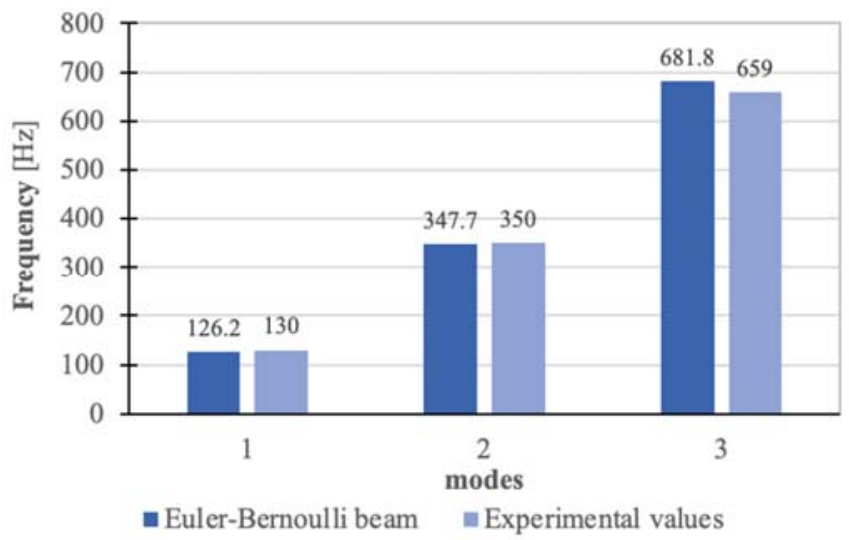

(a)

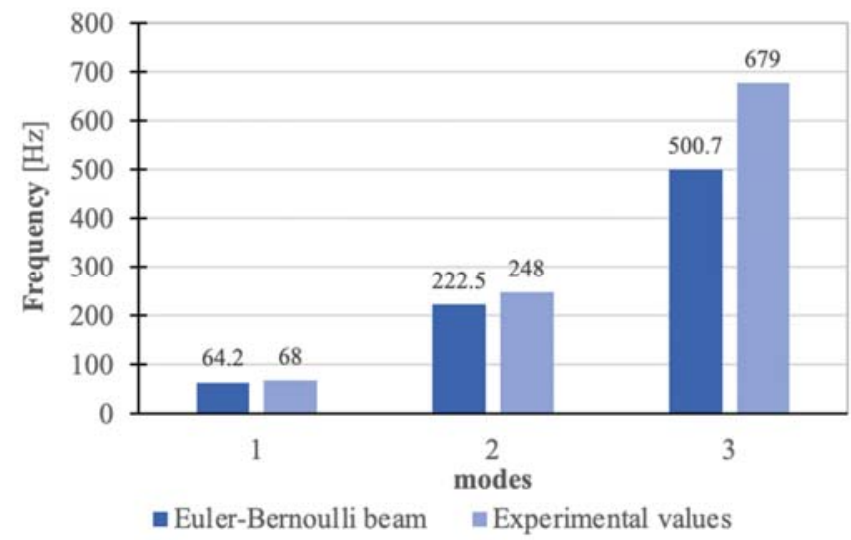

(b)

Figure 9: Theoretical and experimental frequencies for undamaged RC beam B2: (a) free-free ends and (b) hinge-hinge ends.

For each each level of damage by bending (Fig. 10), the RC beam B2, in the un-strengthened condition, was subjected to vibration monitoring. The average frequencies obtained by dynamic tests, for each state of damage Di with $i=1, \ldots, 3$, are summarized in Fig. 11.

After the first phase of concrete's cracking, the damaged RC beam B2 were tested with the presence of a NSM GFRP rod filled into the notch. In this case four damage level were identified: P1 = 4kN - damage degree D1; P2 =8 kN - D2; P3 $=16 \mathrm{kN}-\mathrm{D} 3 ; \mathrm{P} 4=28 \mathrm{kN}$ - D4. In Fig. 12, the crack pattern obtained at the fourth level of damage D4 is shown. Also in this case, after each cycle of loading, modal testing was carried out. Fig. 13 contains the average frequency values measured for strengthened beam B2, considering the first four vibration modes. 


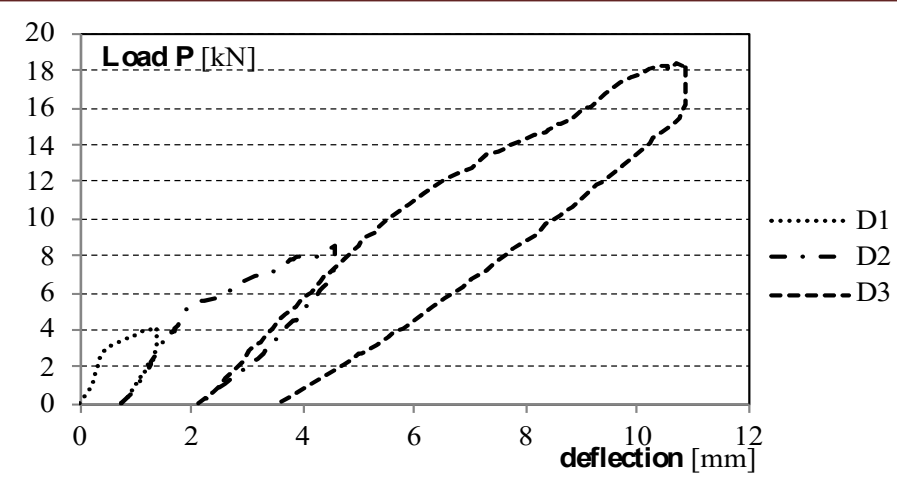

Figure 10: Load-Deflection diagrams for the first three damage level on un-strengthened RC beam B2.

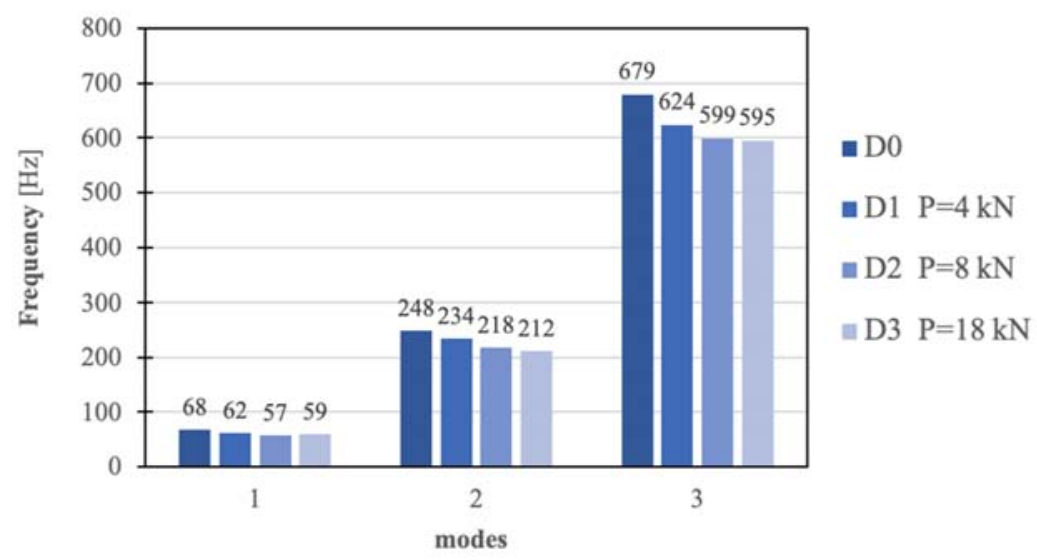

Figure 11: Average frequency values obtained for B2 without strengthening.

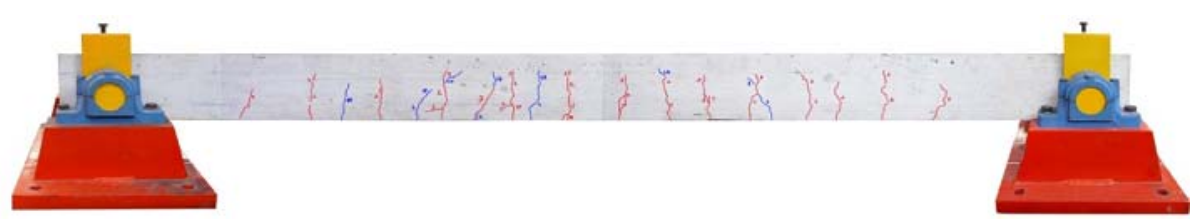

Figure 12: Crack pattern at damage level D4 for beam B2 with NSM GFRP rod.

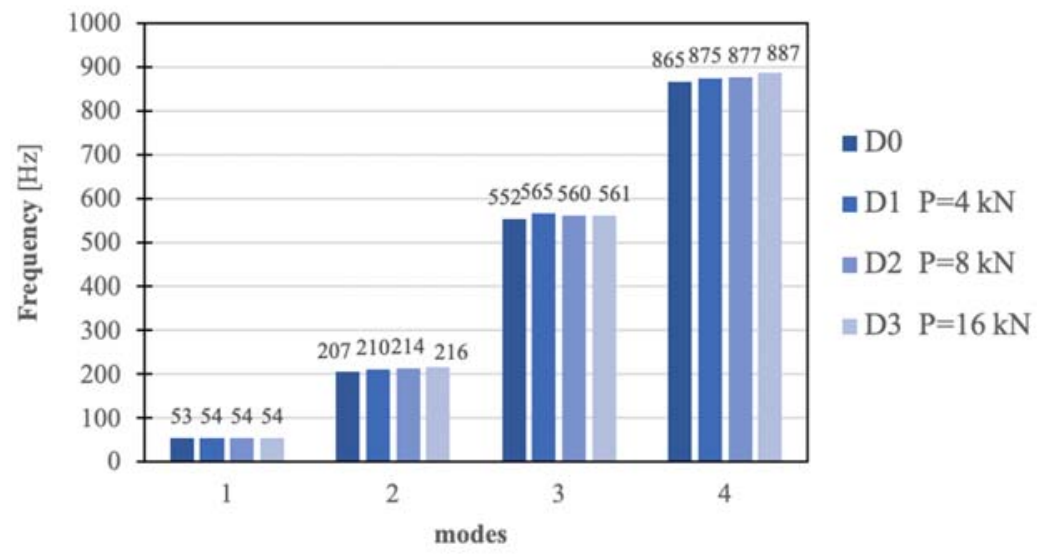

Figure 13: Average frequency values obtained for B2 with NSM GFRP rod strengthening.

For each damage levels, modal testing gives the Frequency Response Functions (FRFs). In Fig. 14, it can be seen the overlap of all FRFs obtain for each state of damage. 


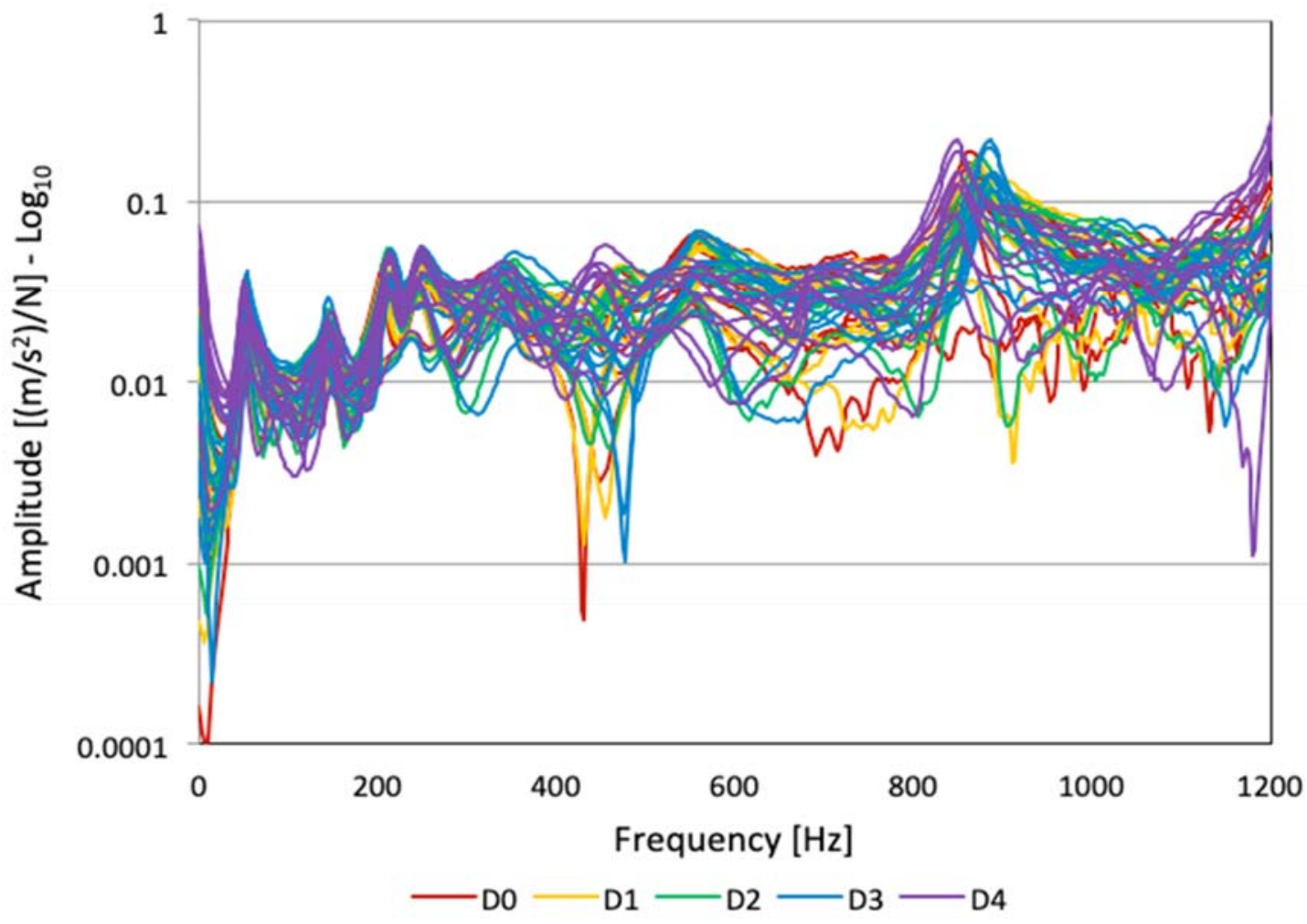

Figure 14: FRFs obtained at each level of damage Di $i=1, \ldots, 4$ for beam B2 strengthened with NSM GFRP rod strengthening.

\section{DISCUSSION OF EXPERIMENTAL RESULTS}

T he results provided by the experimentation allow us to define some useful aspects for the strengthening technique of RC beams according to the NSM method with CFRP and GFRP rods. The influence of the RC beam's nonlinear behavior due to concrete cracking under loading on the beams is reflected in the different vibration modes considered. It can be summarized thus: the variation percent of the frequency values as compared to the integral, nondamaged state, D0, is always increasing from the elastic-linear uncracked phase to the elastic-linear cracked phase, till the inelastic and plastic phase. The natural frequency values tend to describe the beam's global response relative to loss of bending stiffness, and they are less sensitive to local stiffness variations.

Another result that needs to be underlined is the excellent behaviour of the NSM strengthening both with CFRP and GFRP rods in terms of maintaining adherence without exhibiting loss of adherence or damage, with increased resistance capacity of the C-GFRP rod strengthened beams.

Fig. 6 shows for beams B0 and B1, with and without CFRP NSM rods, the comparison between the frequency variations in relation to the average experimental values obtained on undamaged beams, that is on the initial condition for the loading program. It can be noted that the decrease of frequency is more accentuated for beam B0 without strengthening compared to beam $\mathrm{B} 1$ damaged and then strengthened with CFRP rods.

This result is also highlighted in the case of beam B2 damaged and then reinforced with NSM GFRP rod. For the beam without strengthening, the increasing of damage corresponds to the reduction of frequencies, confirmed by variations equal to $10 \% \div 20 \%$ for mode $r=1$ (Fig. 15). The reduction of frequency values at the increment of damage state is less significant for the strengthened beam with NSM GFRP rod (Fig. 16). In this case, the maximum of the frequency variations is equal to about $4 \%$.

The presence of the NSM strengthening with C-GFRP acts by reducing the width of the cracks even for high loads and this is experimentally recorded by the vibrational response of the strengthened beam. 


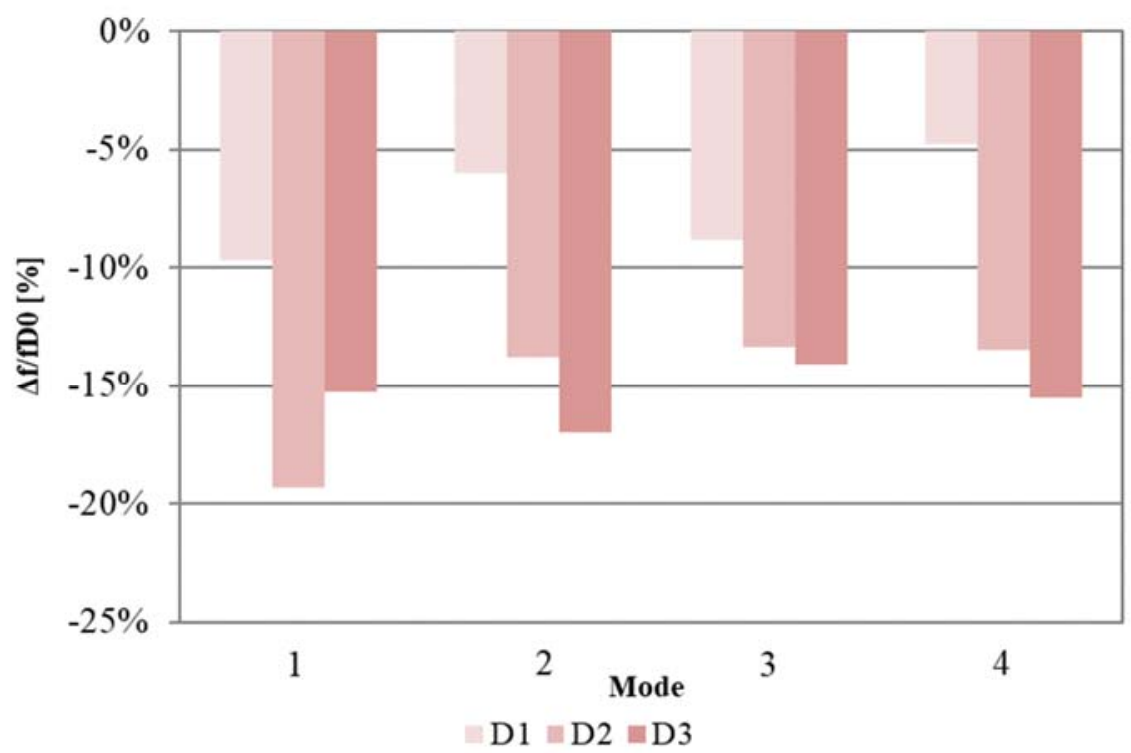

Figure 15: Frequency value variations in percent for the first four modes (beam B2 without strengthening).

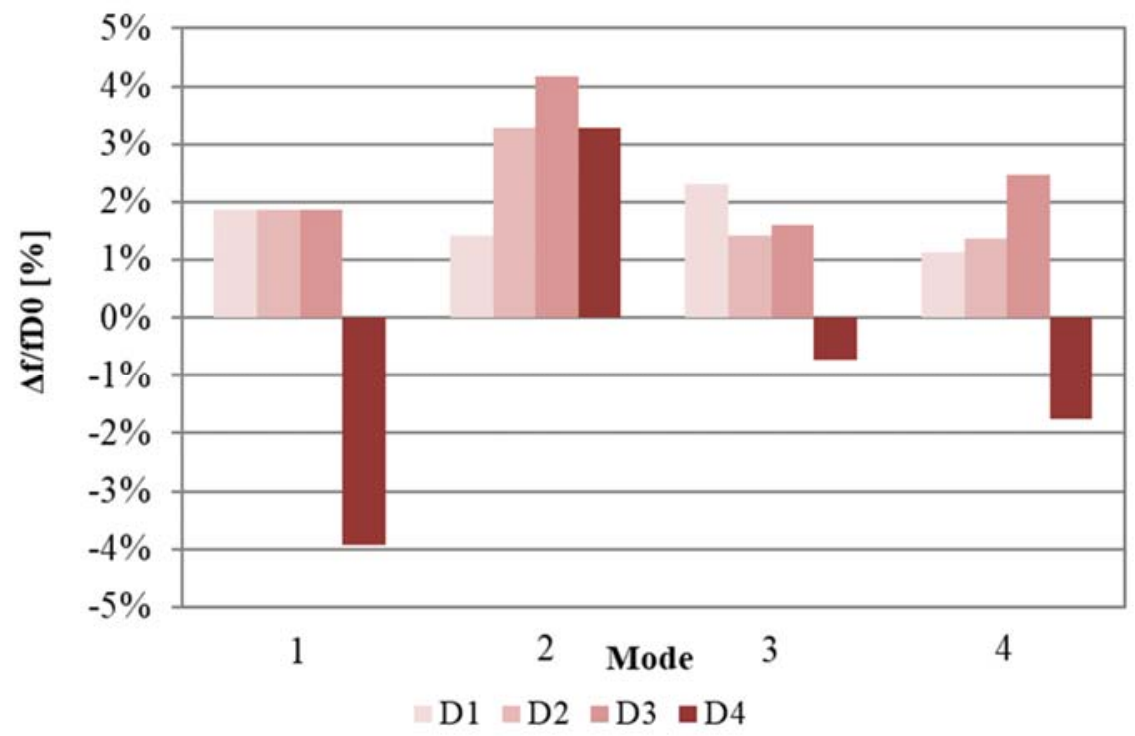

Figure 16: Frequency value variations in percent for the first four modes (beam B2 with NSM GFRP rod).

\section{CONCLUSION}

7 his paper describes an investigation on RC beams strengthened with NSM CFRP and GFRP rods. The investigation foresaw both static and free vibration tests. The main results of the experimental campaign are as follows:

$\checkmark \checkmark$ Excellent behavior of the strengthened beams both with NSM CFRP and NSM GFRP rods in terms of maintaining adherence without exhibiting loss of bond or damage before the collapse of compressive concrete;

$\checkmark$ The presence of the NSM strengthening both with NSM CFRP and NSM GFRP rods acts by reducing the width of the cracks even for high entity of load;

$\checkmark$ The analysis of experimental frequencies is an adequate NDM to monitor RC beams with reinforcement or with strengthening by FRP rods. 


\section{ACKNOWLEDGEMENT}

$\mathrm{T}$ he Polytechnic University of Marche supplied research funds to enable this experimental study. The authors would like to thank all the technicians and students who assisted in the development of this research work.

\section{REFERENCES}

[1] De Lorenzis, L., Teng, J.G. (2007). Near-Surface mounted FRP reinforcement: an emerging technique for strengthening structures, Composites Part B: Engineering, 38(2), pp. 119-143.

[2] Capozucca, R. (2009). Static and dynamic response of damaged RC beams strengthened with NSM CFRP rods, Composite Structures 91(3), 237-248.

[3] El-Hacha, R., Rizkalla, S. (2004). Near-surface mounted fibre reinforced polymer reinforcements for flexural strengthening of concrete structures. ACI Structural Journal, 101(5), pp. 717-726.

[4] Capozucca, R. (2013). Analysis of bond-slip effects in RC beams strengthened with NSM CFRP rods. Composite Structures, 102, pp. 110-123.

[5] Capozucca, R. (2011) Bond-Slip Effects in RC Beams strengthened with Near Surface Mounted CFRP Rods, In: Proceedings Fib Symposium, Prague 2, pp. 779-782.

[6] Cosenza, E., Manfredi, G., Realfonzo, R. (1997). Behavior and modeling of bond of FRP rebars to concrete, ASCE Journal of Composites for Construction, 1(2), pp. 40-51.

[7] De Lorenzis, L., Nanni, A. (2002). Bond between near surface mounted fiber reinforced polymer rods and concrete in structural strengthening. ACI Structural Journal, 99(2), pp. 123-133.

[8] Hassan, T.K., Rizkalla, S. (2004). Bond mechanism of NSM FRP for flexural strengthening of concrete structures, ACI Structural Journal, 101(6), pp. 830-839.

[9] Lees, J.M. (2009). Sensitivity of NSM Reinforcement Forces to Bond-Slip Effects, In: 4th International Conference on Advanced Composites in Construction (ACIC 09), pp. 227-238, Edinburgh.

[10] Perera, K., Ibell, T., Darby, A., Denton, S. (2009). Bond mechanisms of various shapes of NSM CFRP bars. In: 4th International Conference on Advanced Composites in Construction, (ACIC 09), pp. 250-257, Edinburgh.

[11] Lu, X.Z., Teng, J.G., Ye, L.P., Jiang, J.J. (2005). Bond-slip models for FRP sheets/plates bonded to concrete, Engineering Structures, 27(6), pp. 920-937.

[12] Sharaky, I.A., Torres, L., Baena, M., Miàs, C. (2013). An experimental study of different factors affecting the bond of NSM FRP bars in concrete. Composite Structures, 99, pp. 350-365.

[13] De Lorenzis, L., Nanni, A. (2001). Shear strengthening of reinforced concrete beams with NSM fibre-reinforced polymer rods. ACI Struct. J., 98(1), pp. 60-68.

[14] Hassan, T., Rizkalla, S. (2003). Investigation of bond in concrete structures strengthened with near-surface mounted carbon fibre reinforced polymer strips. J. of Comp. for Constr., 7(3).

[15] Sena Cruz, J.M., Barros, J.A.O., Gettu, R., Azevedo, A.F.M. (2006). Bond behavior of near-surface mounted CFRP laminate strips under monotonic and cyclic loading. ASCE, Journal of Composites for Construction, 10, pp. 295-303.

[16] Capozucca, R., Bossoletti, S., Montecchiani, S. (2015). Assessment of RC beams with NSM CFRP rectangular rods damaged by notches. Composite Structures, 128, pp. 322-341.

[17] Blaschko M. (2003). Bond behaviour of CFRP strips glued into slits. In: Proceedings FRPRCS-6. Singapore: World Scientific, pp: 205-214.

[18] Nanni, A., Di Ludovico, M., Parretti, R. (2004). Shear Strengthening of a PC Bridge Girder with NSM CFRP Rectangular Bars. Advances in Structural Engineering, 7(4), pp. 297-309.

[19] Issa, M.S., Metwally, I.M., Elzeiny, S.M. (2011). Influence of fibres on flexural behavior and ductility of concrete beams reinforced with GFRP rebars. Eng. Structures, 33, pp.1754-1763.

[20] Capozucca, R. (2014). On the strengthening of RC beams with near surface mounted GFRP rods. Composite Structures, 117C, pp: 143-155.

[21] Ding, Y., Ning, X., Zhang, Y., Pacheco-Torgal, F., Aguiar, J.B. (2014). Fibres for enhancing of the bond capacity between GFRP rebar and concrete. Construction and Building Materials, 51, pp: 303-312.

[22] Masmoudi, A., Ouezdou, M., Ben, Bouaziz J. (2012). New parameter design of GFRP RC beams. Construction and Building Materials, 29, pp. 627-632. 
[23] Capozucca, R. (2018). Vibration analysis of damaged RC beams strengthened with GFRP. Composite Structures, 200, pp. 624-634.

[24] Capozucca, R., Bossoletti, S. (2014). Static and free vibration analysis of RC beams with NSM CFRP rectangular rods. Composites Part B: Engineering, 67, pp. 95-110.

[25] ASTM D 3039/D 3039 M - 08. Standard Test Method for Tensile Properties of Polymer Matrix Composite Materials. American Standard of Testing and Materials, (2008). 